\title{
Post-Transcriptional Control of Angiotensin II Type 1 Receptor Regulates Osteosarcoma Cell Death
}

\author{
Yue Zhao ${ }^{\mathrm{a}}$ Kaicheng $\mathrm{Xu}^{\mathrm{b}}$ Peng Liuc \\ aDepartment of Vascular Surgery, China-Japan Union Hospital, Jilin University, Changchun, ${ }^{b}$ Department \\ of Anesthesiology, China-Japan Union Hospital, Jilin University, Changchun, 'Department of \\ Orthopaedics, China-Japan Union Hospital, Jilin University, Changchun, China
}

\section{Key Words}

Osteosarcoma (OS) • Angiotensin II type 1 receptor (AGTR1) • MiR-1248 • Chemotherapy

\begin{abstract}
Background/Aims: MicroRNAs (miRNAs) play an essential role in the tumorigenesis of osteosarcoma (OS). However, the effects of miR-1248 on chemo-resistant potential of OS have not been studied. Here, we addressed this question. Methods: The levels of miR-1248 and apoptotic protein angiotensin II type 1 receptor (AGTR1) in OS specimens were examined by RT-qPCR and Western blotting, respectively. The relationship between miR-1248 and AGTR1 was determined by analysis of Spearman's Rank Correlation Coefficients. The patient survival was determined with Kaplan-Meier curves. Bioinformatics analyses were done to predict microRNAs (miRNAs) that target AGTR1. The functional binding of miRNAs to AGTR1 mRNA was examined by a dual luciferase reporter assay. Cell viability was determined by an CCK- 8 assay. Apoptosis was determined by a fluorescence-based apoptosis assay. Results: The levels of miR-1248 were significantly elevated while the levels of AGTR1 were significantly decreased in OS specimens than in paired adjacent normal tissue. The levels of miR-1248 were negatively correlated to the levels of AGTR1. Moreover, the patients with high miR-1248 levels had poorer survival than those with low MiR-1248 levels, and the patients with low AGTR1 levels had poorer survival than those with high AGTR1 levels. MiR-1248 inhibited protein translation of AGTR1, through binding to the 3'-UTR of the AGTR1 mRNA. The AGTR1-mediated cell apoptosis was suppressed by overexpressing miR-1248, and was augmented by depleting miR-1248. Conclusion: Increased miR-1248 expression in OS may inhibit AGTR1-mediated cancer cell death in chemotherapy. The outcome of chemotherapy may be improved by the suppression of miR-1248 in OS cells.

\section{Introduction}

Osteosarcoma (OS) is one of the most malignant bone tumor. The prognosis of OS is generally bad for the distal metastases [1-3]. In nowadays, the prognosis of patients with early 
OS could be improved with chemotherapy. However, the prognosis for OS patients remains poor even with the help of chemotherapy because some OS are resistant to chemotherapy and radiation therapy [4-6]. Thus, great efforts have been made to elucidate the mechanisms underlying the invasion and chemo-resistance of OS [7-10]. In specific conditions, the bad sensitivity towards a certain chemotherapy may be resulted from the enhanced antiapoptotic potentials of the cancer cells [11-13]. Cellular apoptosis is regulated by certain proteins, which are apoptosis activating proteins, e.g. Bid, Bak, Bad, and angiotensin II type 1 receptor (AGTR1) [14, 15].

AGTR1 mediates the major cardiovascular effects of angiotensin II, which has vasopressor effects and regulates aldosterone secretion. AGTR1 is involved in tumorigenesis of many cancers. Losartan has been effective in cancer treatment for the inhibition of AGTR1 [16-18]. However, the role of AGTR1 in tumorigenesis appears to be cancer-type specific. Although AGTR1 has been determined as an oncogene in breast cancer, recent reports suggest different role of AGTR1 in other cancers. For example, Pu et al. recently showed that miR-34a-5p promotes the multi-chemoresistance of osteosarcoma via modulating AGTR1, which negatively correlates with osteosarcoma chemoresistance [19]. Down-regulation of the AGTR1 expression suppresses cell apoptosis, while over-expression of AGTR1 promotes the drug-triggered cell death [19].

MicroRNAs (miRNAs) is a class of non-coding small RNA with the length of about 18-23 nucleotides. Past studies have demonstrated the regulation effects of miRNAs on protein expression. MiRNAs combine with 3'-untranslated region (3'-UTR) of the mRNA of certain protein thus regulating the intracellular protein expression [20-24]. Many biological events including development of a tumor would be regulated by miRNAs [20-24]. In addition, the aberrant expression of miRNAs would contribute to OS initiation, progression, outgrowth, metastases, as well as resistance to chemotherapy [20-24]. MiR-1248 is a rarely studied miRNA, and so far no reports have addressed to its biological function.

In this study, we studied the effects of miR-1248 on OS cell survival during chemotherapy.

\section{Materials and Methods}

\section{OS patient specimens}

The OS tissue specimens and corresponding adjacent non-tumor bone tissue (NT) were obtained from OS patients during surgery. The patients were histologically and clinically diagnosed as OS at the ChinaJapan Union Hospital (Table 1). This study was approved from the Research Ethics Committee of the ChinaJapan Union Hospital and informal consents were signed by all patients.

Cell line culture and treatment

The OS cells applied in this study was U2OS cell line (ATCC, Rockville, MD, USA). It was derived from a moderately differentiated sarcoma of the tibia, which was a representative human os cell line. U2OS cell line were cultured in Dulbecco's Modified Eagle's Medium (DMEM, Invitrogen, USA) supplemented with $10 \%$ fetal bovine serum (FBS, SigmaAldrich, USA). The cells were cultured at $37{ }^{\circ} \mathrm{C}$ with $5 \% \mathrm{CO}_{2}$ in an incubator. In cell viability test, U20S cells were treated with 10 $\mu \mathrm{mol} / \mathrm{l}$ of Fluorouracil (5-FU; Sigma-Aldrich).

Table 1. Clinical-pathological characteristics (total)

\begin{tabular}{lc}
\hline & Patients (n; \%) \\
\hline OS tissue/ Normal tumor-adjacent tissue & $40(100 \%) / 40(100 \%)$ \\
Age $(<60 / \geq 60$ years old) & $20(50 \%) / 20(50 \%)$ \\
Gender (male/female) & $24(60 \%) / 16(40 \%)$ \\
Tumor site (bone) & $40(100 \%)$ \\
Tumor grade (well or moderate/poor) & $16(40 \%) / 12(30 \%) / 12(30 \%)$ \\
Tumor size (T1/T2/T3/T4) & $12(30 \%) / 13(32.5 \%) / 12(30 \%) / 3(7.5 \%)$ \\
Lymph node metastasis (no/yes) & $32(80 \%) / 8(20 \%)$ \\
Distal metastasis (no/yes) & \\
\hline
\end{tabular}




\section{Cellular Physiology Cell Physiol Biochem 2018;45:1581-1589

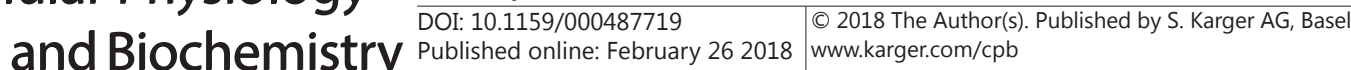 \\ Zhao et al.: Modulation of OS Chemo-Sensitivity}

\section{Transfection}

MiR-1248-modulating plasmids composed of a backbone plasmid and a GFP reporter (pcDNA3.1-CMVGFP, Clontech, USA). The miR-1248 mimic, antisense and control null were all synthesized (RiboBio Co., Ltd. Guangdong, China). All these miRNAs were subcloned into a pcDNA3.1-CMV-GFP backbone. The small 2A peptide sequences were applied. The plasmid was sequenced for ensuring the correct structure. Then the new plasmids were transfected with Lipofectamine 2000 (Invitrogen, USA). Per general specifications, there would be co-expressed GFP after success transfection. Thus, the cells with effective transfection were purified with flow cytometry (FACScan, BD, USA).

\section{Western blot}

The protein was analyzed with western blot. For the tissues, the total protein was extracted after homogenizing. The total proteins were extracted per manufacture's introduction (Sigma, USA). All the steps were manipulated on ice for minimizing degradation. The concentration of extracted total protein was determined with BCA protein assay kit (Bio-rad, China). Next, the total protein was electrophoresed with SDS-polyacrylamide gels after heating for $5 \mathrm{~min}$ at $100{ }^{\circ} \mathrm{C}$. The separated proteins on SDS gels were then transferred on a PVDF membrane. After blocking with 5\% FBS, the antibodies against target proteins (rabbit anti-AGTR1 and anti- $\alpha$-tubulin, Cell Signaling, USA) were applied and incubated with the PVDF membrane at $4^{\circ} \mathrm{C}$ overnight. After washing, the HRP-conjugated anti-rabbit (Jackson ImmunoResearch Labs, USA) was applied and incubated at $37^{\circ} \mathrm{C}$ for 1 hour. The membrane was developed with the enhanced chemiluminescent system and imaged with X-ray film. The images were quantified with ImageJ (NIH, USA). The $\alpha$-tubulin was applied as internal control. The protein levels were normalized to the level of $\alpha$-tubulin and then compared to the levels of experimental controls. The experiment was repeated for five times.

\section{Quantitative RT-PCR}

Total mRNA was extracted from tissue specimens or cells lines. For the tissues, the mRNA was extracted after homogenizing. For cell lines, the mRNA was extracted after lysis. The total mRNA was extracted per manufacturer's introductions with miRNeasy mini kit (Qiagen, Germany). Complementary DNA (cDNA) was obtained from $2 \mu \mathrm{g}$ of total RNA with High-Capacity cDNA Reverse Transcription Kit (Applied Biosystems, USA). The primers to target RNA were purchased from Qiagen for further RT-qPCR (QuantiTect SYBR Green PCR Kit, Qiagen, USA). Data were collected and analyzed for quantifying the relative expression levels of mRNA. The $\alpha$-tubulin was applied as an internal control. The experiment was repeated thrice.

\section{3'-UTR luciferase-reporter assay}

The algorithm TargetSan was applied for predicting the miRNAs targets (https://www.targetscan.org). The 3'-UTR reporter plasmids were purchased from Creative Biogene (Shirley, USA), including an AGTR1 3'-UTR reporter plasmid (pRL-AGTR1 3'-UTR) and an AGTR1 3'-UTR reporter plasmid with a mutant at the miR-1248 binding site (pRL-AGTR1 3'-UTR mut). The U2OS cell line were cultured to obtain a certain density $\left(5 \times 10^{4}\right.$ cells per well). Then the cells were co-transfected with pRL-AGTR1 3'-UTR or pRL-AGTR1 3'-UTR mut by Lipofectamine 2000, as well as miR-1248, miR-1248 antisense or miR-1248 null. After cotransfection, the cells were collected for further assay. The cells were analyzed with dual-luciferase reporter gene assay kit (Promega, China), per the manufacturer's instructions.

\section{In vivo implantation of tumor cells and quantification of tumor size}

MiR-1248-overexpressing and control U2OS cells (106; miR-1248/null) were subcutaneously injected under the skin at the back of the NOD/SCID mice. The tumors were allowed to grow for 1 month before dissecting out for quantification of the weight.

Cell counting kit-8 (CCK-8) assay

The cell viability after chemotherapy treatment was determined with CCK-8 assay (Sigma, USA). The cells were cultured in 96-well plate to obtain a density of $5 \times 10^{4} / \mathrm{ml}$. Then the cells were treated with 5 -FU or control for $24 \mathrm{~h}$. Then CCK-8 solution (20ml/well) was added in the wells with cells treated with resveratrol or control and incubated at $37^{\circ} \mathrm{C}$ for $2 \mathrm{~h}$. The optical density (OD) value was read with microplate reader at $450 \mathrm{~nm}$. Then the cell viability would be calculated as: the OD value of treated wells/control group*100\%. 


\section{Cellular Physiology Cell Physiol Biochem 2018;45:1581-1589 \begin{tabular}{ll|l} 
and Biochemistry Published online: February 262018 & $\begin{array}{l}\text { (c) } 2018 \text { The Author(s). Published by S. Karger AG, Basel } \\
\text { www.karger.com/cpb }\end{array}$ \\
\hline
\end{tabular}

Apoptosis assay by flow cytometry

The cells after treatment were re-suspended in PBS to obtained a cell density of $10^{6} / \mathrm{ml}$. The cells were stained with Apoptosis Detection Kit I (BD, USA), including both the FITC-Annexin V and propidium iodide (PI). Cells after staining were analyzed with flow cytometer (FACScan, BD, USA). The results were interpreted with Cell Quest software.

\section{Statistical analysis}

All data were analyzed using one-way ANOVA with Bonferroni correction. The data of different groups were compared with Fisher's Exact Test (GraphPad Prism, GraphPad Software, Inc. USA). Spearman's Rank Correlation Coefficients were also involved to obtain the bivariate correlations. The patient survival was demonstrated with Kaplan-Meier curves. All the data values were expressed as mean \pm standard deviation (SD). When $\mathrm{p}<0.05$, it was considered as significant.

\section{Results}

The increased miR-1248 level and decreased AGTR1 level in OS specimens

For the OS specimens from 40 patients, the miR-1248 level was significantly higher than that of in NT tissues (Fig. 1). Conversely, the AGTR1 level was significantly lower (Fig .1). In addition, the level of miR-1248 was negatively correlated with the levels of AGTR1, which was significantly (Fig $1, \gamma=-0.73, \mathrm{p}<0.0001$ ). Thus, it suggested that there may be a direct and reverse relationship between the levels of miR-1248 and AGTR1 in clinical OS cells.

The relation between miR-1248 or AGTR1 level and overall survival of OS patients was also analyzed. For the 40 patients in this study, the median value of miR-1248 or AGTR1 levels was applied as a cutoff. The patients with miR-1248 or AGTR1 level higher than cutoff were classified as miR-1248-high/AGTR1-high cases $(\mathrm{n}=20)$ and patients with miR-1248 or AGTR1 level lower than cutoff were classified as miR-1248-low/AGTR1-low cases ( $n=20)$. The survival of all patients was recorded in a 5-year follow up. Kaplan-Meier curves were plotted with the miR-1248 or AGTR1 levels. For OS patients involved in this study, the result indicated a poorer overall survival in miR-1248-high cases than that of in miR-1248-low cases (Fig. 1D), and a poorer overall survival in AGTR1-low cases than that of in AGTR1-high cases (Fig. 1E).

The inhibited translation of AGTR1 mRNA with miR-1248 in OS cells

Above result has proved a negative correlation between the level of miR-1248 and AGTR1 in OS cells. We have tried to explore the potential mechanism. From the prediction of bioinformatics analyses, miR-1248 was indicated to bind the 3'-UTR of AGTR1 mRNA ranged from $566^{\text {th }}$ to $573^{\text {th }}$ base site (Fig. 2A). The translation of AGTR1 mRNA may be inhibited after binding with miR-1248 thus suppressing the expression of AGTR1 protein.

The prediction was confirmed to figure out the effects of miR-1248 on AGTR1 mRNA in OS cells. The miR-1248 was over-expressed or depleted in U2OS cells by transfecting the cells with plasmids carrying miR-1248-mimic (miR-1248), a miR-1248 antisense (asmiR-1248), or null sequence as a control (null). The miR-1248 levels in U2OS cells after overexpression or depletion were determined by RT-qPCR (Fig. 2B). Then the transfected U20S cells were transfected with either plasmids carrying luciferase reporter for 3'-UTR of AGTR1 mRNA, or plasmids carrying luciferase reporter for 3'-UTR of AGTR1 mRNA with one mutate at the miR-1248 binding site (mut). From the luciferase activities, in the U2OS cells with over-expressed miR-1248, the 3'-UTR of AGTR1 mRNA was specifically combined, thus the translation of AGTR1 mRNA was inhibited (Fig. 2C).

The reduced AGTR1 protein in OS cells

From the result, the levels of AGTR1 mRNAwere notvaried in U2OS cells with differentmiR1248 levels (Fig. 3A). However, the levels of AGTR1 protein in the U2OS cells were significantly related to miR-1248 levels. Specifically, AGTR1 protein were significantly decreased with 
Fig. 1. Increased miR-1248 and decreased AGTR1 are correlated in OS. RT-qPCR on miR-1248 and Western blot for AGTR1 were performed on 40 paired OS and the adjacent non-tumor bone tissues (NT). (A) AGTR1 levels. (B) miR1248 levels. (C) A Correlation test between AGTR1 and miR-1248. (D) The 40 patients were followed-up for 5 years. The median value of all 40 cases was chosen as the cutoff point for separating miR-1248high cases $(n=20)$ from miR-1248low cases $(n=20)$. Kaplan-Meier curves were performed to evaluate the overall survival of the OS patients, based on miR-1248 levels. (E) The 40 patients were followedup for 5 years. The median value of all 40 cases was chosen as the cutoff point for separating AGTR1high cases $(n=20)$ from AGTR1-low cases $(n=20)$. Kaplan-Meier curves were performed to evaluate the overall survival of the OS patients, based on AGTR1 levels. ${ }^{*} p<0.05$. $* * \mathrm{p}<0.01 . \mathrm{N}=40$.

Fig. 2. MiR-1248 targets 3 'UTR of AGTR1 mRNA to inhibit its expression. (A) Bioinformatics analyses showing binding of miR-1248 to the 3'-UTR of AGTR1 mRNA. (B) We either overexpressed miR-1248, or inhibited miR-1248 in a human OS cell line, U2OS, by transfection of the cells with a miR-1248expressing plasmid (miR-1248), or with a plasmid carrying miR-1248 antisense (as-miR-1248). The U20S cells were also transfected with a plasmid carrying a null sequence as a control (null). The modification of miR-1248 levels in U2OS cells was confirmed by RT-qPCR. (C) MiR-1248-modified U20S cells were then transfected with $1 \mu \mathrm{g}$ plasmids carrying luciferase reporter for 3'-UTR of AGTR1 mRNA. Moreover, null-transfected U2OS cells were also transfected with $1 \mu \mathrm{g}$ plasmids carrying luciferase reporter for 3'-UTR of AGTR1 mRNA with one mutate at the miR-1248 binding site (mut). The luciferase activities were examined. ${ }^{*} \mathrm{p}<0.05$. $\mathrm{N}=5$.

over-expressed miR-1248 while it was significantly increased after the depletion of miR1248 (Fig. 3B). It concluded that miR-1248 inhibited translation of the AGTR1 mRNA into
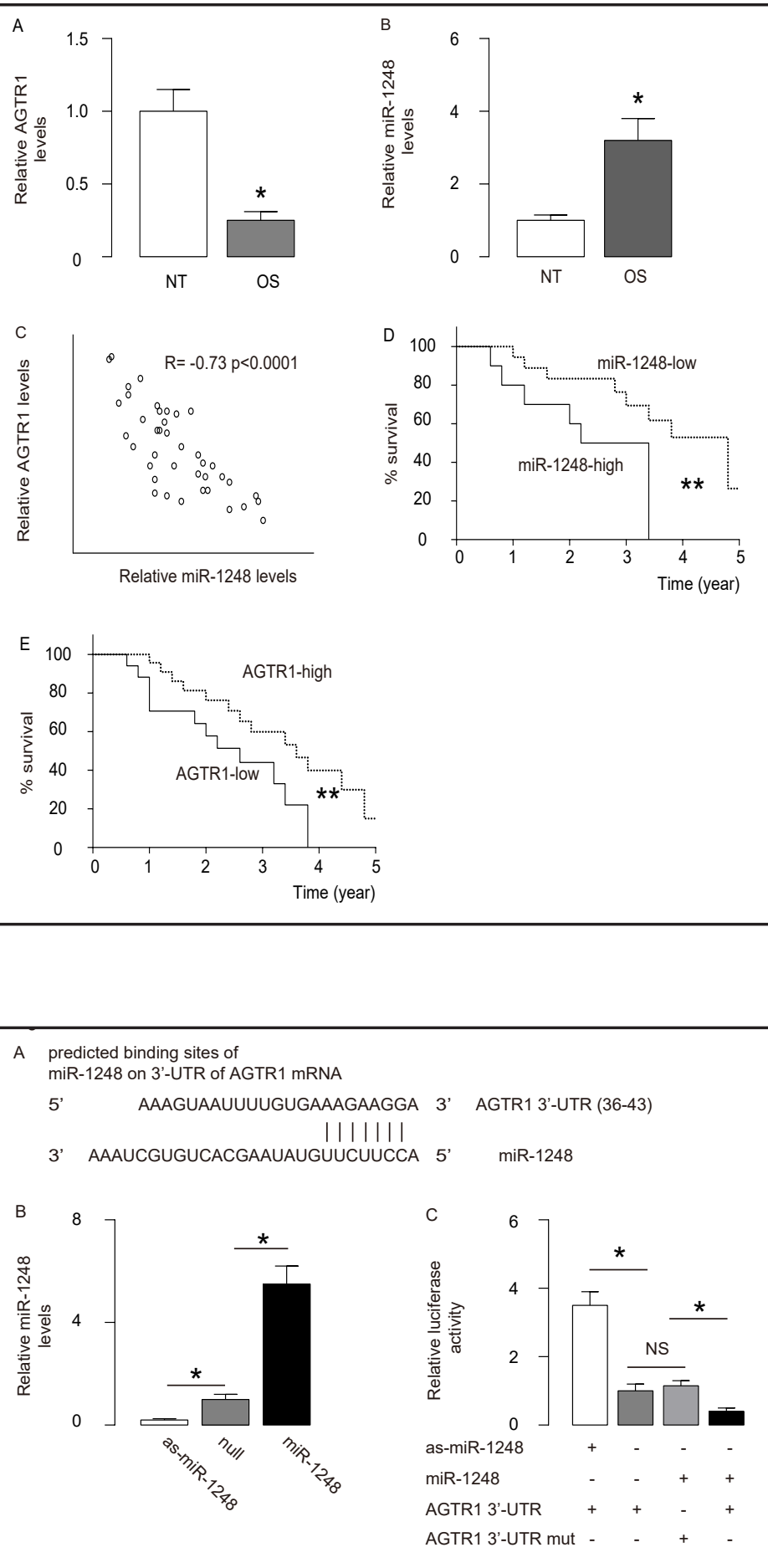
protein in OS cells, instead of suppressing the levels of AGTR1 mRNA.

The increased cell viability in $5-F U$ treated OS cells and decreased cell apoptosis with miR1248

The effects of miR1248 on both cell viability after 5-FU treatment and cell apoptosis were explored. The U2OS cells with different expression levels of miR-1248 were treated with 5-FU and the cell viability were analyzed with an CCK-8 assay. It observed that the U2OS cells viability after 5-FU treatment was increased with overexpressed miR1248, while the U2OS cells viability after 5-FU treatment was decreased with the depletion of miR-1248 (Fig. 4A). In addition, the apoptosis assay was also performed and quantified. It found that U20S cell apoptosis would be decreased with overexpressed miR-1248, while it would be increased with the depletion of miR-1248 (Fig. 4B and 4C). Hence, it suggested that 5-FU-induced OS cell apoptosis may be lower with higher levels of miR-

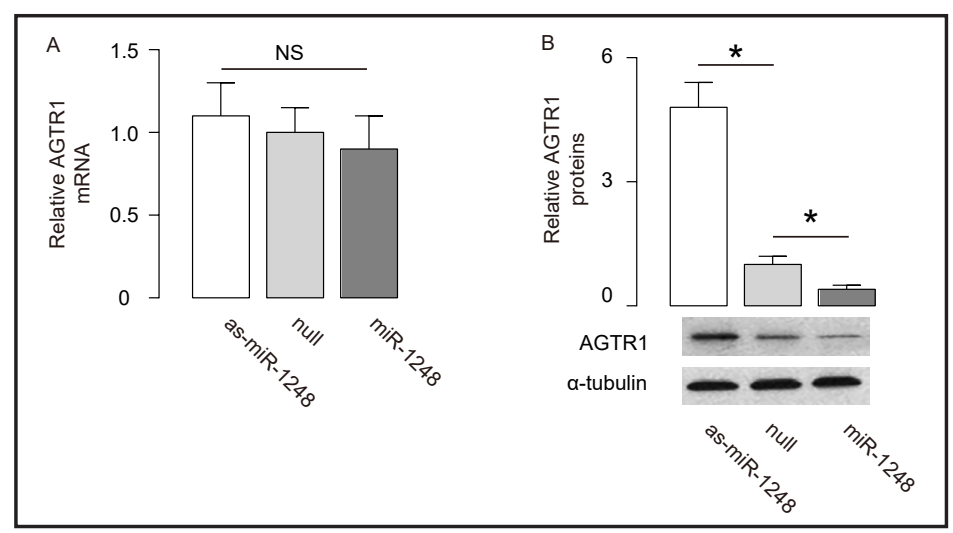

Fig. 3. MiR-1248 decreases AGTR1 protein, but not mRNA, in OS cells. (A-B) The AGTR1 mRNA levels (A) and protein levels (B) in miR-1248modified U20S cells. ${ }^{*} \mathrm{p}<0.05$. NS: non-significant. $\mathrm{N}=5$.

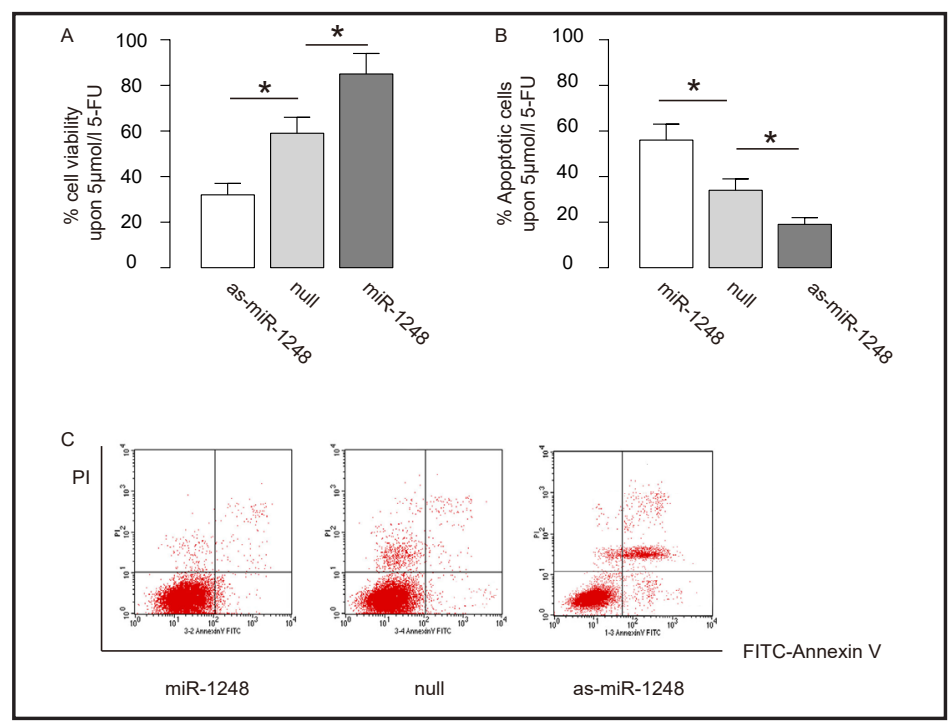

Fig. 4. MiR-1248 increases 5-FU-induced OS cell apoptosis. (A) The effects of miR-1248 modification on OS cell viability at presence of $5 \mu \mathrm{mol} / \mathrm{l}$ 5-FU in an CCK-8 assay. (B-C) Fluorescence-based apoptosis assay on miR-1248-modified U2OS cells, shown by quantification (B), and by representative flow charts (C). ${ }^{*} \mathrm{p}<0.05 . \mathrm{N}=5$.

1248 and it may also be related to the chemo-resistance of clinical OS cells.

\section{Increased OS cell growth with miR-1248 in vivo}

The tumor growth of OS cells with different levels of miR-1248 was evaluated in vivo. The miR-1248-modified U2OS cells (miR-1248) or control cells (null) were implanted into NOD/SCID mice at a density of $10^{6}$ cells. One month after tumor cell transplantation, the tumor was removed and weighed. The weight of miR-1248-overexpressing U2OS -cellformed tumor was significantly higher than control, shown by gross images (Fig. 5A and 5B). These results suggest that OS cell survival during chemotherapy would be attributable to the increased expression of miR-1248, in which the chemotherapy-induced cell apoptosis could be attenuated via miR-1248-mediated suppression of AGTR1 protein. Thus, it is anticipated that chemotherapy-induced OS cell apoptosis may be enhanced after miR-1248 inhibition, resulting in improved patient prognosis and survival. 


\section{Cellular Physiology Cell Physiol Biochem 2018;45:1581-1589

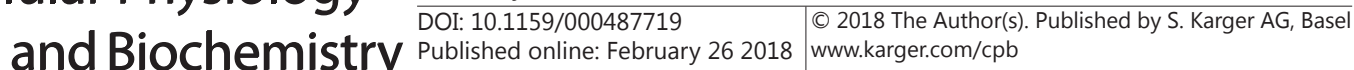

\section{Discussion}

MiRNAs participate in the regulation of gene expression after transcription. Many previous studies have reported the effects of various miRNAs and most of the studies have focused on the functions of miRNAs on the tumorigenesis, development, metastases and treatment. OS is a malignant tumor with complicated pathogenesis. It reported that miRNAs can make effects in OS from many pathways, both direct function and indirect coordination with other factors [22]. There was a lack of effective treatment for OS. The study of miRNAs in OS tumorigenesis and pathogenesis may help to demonstrate the mechanism and suggest potential target for OS treatment.

The chemo-therapy resistance leads to a bad prognosis of OS. The activation of anti-apoptotic pathway against chemotherapeutics contributed to the resistance of OS cells. The apoptosis of OS cells was inhibited. A previous study has focused on the apoptosis inhibition of OS cells and AGTR1 was found to be a key factor [19]. In the 5-FU induced apoptosis in OS cells, it observed the activation of many apoptosis-associated proteins, such as AGTR1, Bid and Bak. All these proteins would lead to the initiation of apoptosis process by upregulating the Cytochrome $\mathrm{C}$ and caspases. However, the effects of chemotherapy could also be suppressed with 5-FU by inhibiting AGTR1 in OS cells.

The candidate miRNAs with the function of AGTR1-targeted inhibitor were predicted with bioinformatics analyses. All the predicted miRNAs were screened and determined in OS specimens. MiR-1248 was significantly increased in OS specimens compared to corresponding normal tissues. Thus, miR-1248 was assumed to make effects in OS cells by targeting AGTR1. Further study confirmed the correlation test between miR-1248 and AGTR1 in OS specimens. In addition, the mechanism was also explored. The 3'-UTR of AGTR1 mRNA would be combined with miR-1248, leading to down-regulated translation of AGTR1 protein. The miR-1248 levels were also over-expressed or depleted in OS cells and it observed that the level of AGTR1 mRNA was not significantly varied. Above results proved than the high miR-1248 level would down-regulate the expression level of AGTR1 protein by suppressing the AGTR1 mRNA translation instead of down-regulating AGTR1 mRNA.

The effects of miR-1248 on OS cells were also explored. The cell viability of 5-FU treated OS cell seemed to be enhanced with over-expressed miR-1248. It may be resulted from the miR-1248-mediated decrease of AGTR1 protein. Similar results have been observed in other OS cells lines, e.g. SaOS-2, further confirming our results were not specific to certain cell line.

In this study, a potential mechanism was demonstrated for interpreting the chemotherapy resistance of OS cells. The miR-1248 expressed in OS cells inhibited the translation of AGTR1 protein. The cell viability of OS cells would be enhanced thus suppressing the cell apoptosis towards chemotherapy. The miR-1248 may be a new target against chemotherapy resistance in OS cells. 


\section{Cellular Physiology Cell Physiol Biochem 2018;45:1581-1589 \begin{tabular}{ll|l} 
and Biochemistry Published onlıne: February 262018 & $\begin{array}{l}\text { C } 2018 \text { The Author(s). Published by S. Karger AG, Basel } \\
\text { www.karger.com/cpb }\end{array}$ \\
\hline
\end{tabular} \\ Zhao et al.: Modulation of OS Chemo-Sensitivity}

\section{Disclosure Statement}

No conflict of interests exists.

\section{References}

1 Zhang N, Xie T, Xian M, Wang YJ, Li HY, Ying MD, Ye ZM: SIRT1 promotes metastasis of human osteosarcoma cells. Oncotarget 2016;7:79654-79669.

-2 Gozo MC, Jia D, Aspuria PJ, Cheon DJ, Miura N, Walts AE, Karlan BY, Orsulic S: FOXC2 augments tumor propagation and metastasis in osteosarcoma. Oncotarget 2016;7:68792-68802.

-3 Cam M, Gardner HL, Roberts RD, Fenger JM, Guttridge DC, London CA, Cam H: DeltaNp63 mediates cellular survival and metastasis in canine osteosarcoma. Oncotarget 2016;7:48533-48546.

-4 Ferrari S, Serra M: An update on chemotherapy for osteosarcoma. Expert Opin Pharmacother 2015;16:2727-2736.

5 Xiao X, Wang W, Wang Z: The role of chemotherapy for metastatic, relapsed and refractory osteosarcoma. Paediatr Drugs 2014;16:503-512.

6 Yang C, Yang QO, Kong QJ, Yuan W, Ou Yang YP: Parthenolide Induces Reactive Oxygen Species-Mediated Autophagic Cell Death in Human Osteosarcoma Cells. Cell Physiol Biochem 2016;40:146-154.

7 Hattinger CM, Biason P, Iacoboni E, Gagno S, Fanelli M, Tavanti E, Vella S, Ferrari S, Roli A, Roncato R, Giodini L, Scotlandi K, Picci P, Toffoli G, Serra M: Candidate germline polymorphisms of genes belonging to the pathways of four drugs used in osteosarcoma standard chemotherapy associated with risk, survival and toxicity in non-metastatic high-grade osteosarcoma. Oncotarget 2016;7:61970-61987.

-8 Huang Y, Liu B, Sun Y, Zhang J, Yao Y, He A: The prognostic value of D-dimer levels in metastatic osteosarcoma patients treated with second-line chemotherapy. Oncotarget 2016;7:65568-65576.

-9 Wang L, Yang L, Lu Y, Chen Y, Liu T, Peng Y, Zhou Y, Cao Y, Bi Z, Liu T, Liu Z, Shan H: Osthole Induces Cell Cycle Arrest and Inhibits Migration and Invasion via PTEN/Akt Pathways in Osteosarcoma. Cell Physiol Biochem 2016;38:2173-2182.

$>10$ Li Y, Wagner ER, Yan Z, Wang Z, Luther G, Jiang W, Ye J, Wei Q, Wang J, Zhao L, Lu S, Wang X, Mohammed MK, Tang S, Liu H, Fan J, Zhang F, Zou Y, Song D, Liao J, Haydon RC, Luu HH, He TC: The Calcium-Binding Protein S100A6 Accelerates Human Osteosarcoma Growth by Promoting Cell Proliferation and Inhibiting Osteogenic Differentiation. Cell Physiol Biochem 2015;37:2375-2392.

11 Roos A, Satterfield L, Zhao S, Fuja D, Shuck R, Hicks MJ, Donehower LA, Yustein JT: Loss of Runx2 sensitises osteosarcoma to chemotherapy-induced apoptosis. Br J Cancer 2015;113:1289-1297.

12 Hu W, Xiao Z: Formononetin induces apoptosis of human osteosarcoma cell line U2OS by regulating the expression of Bcl-2, Bax and MiR-375 in vitro and in vivo. Cell Physiol Biochem 2015;37:933-939.

13 Hu KH, Li WX, Sun MY, Zhang SB, Fan CX, Wu Q Zhu W, Xu X: Cadmium Induced Apoptosis in MG63 Cells by Increasing ROS, Activation of p38 MAPK and Inhibition of ERK 1/2 Pathways. Cell Physiol Biochem 2015;36:642-654.

14 Kooptiwut S, Hanchang W, Semprasert N, Junking M, Limjindaporn T, Yenchitsomanus PT: Testosterone reduces AGTR1 expression to prevent beta-cell and islet apoptosis from glucotoxicity. J Endocrinol 2015;224:215-224.

15 Plummer S, Tower C, Alonso P, Morgan L, Baker P, Broughton-Pipkin F, Kalsheker N: Haplotypes of the angiotensin II receptor genes AGTR1 and AGTR2 in women with normotensive pregnancy and women with preeclampsia. Hum Mutat 2004;24:14-20.

-16 de Ronde JJ, Lips EH, Mulder L, Vincent AD, Wesseling J, Nieuwland M, Kerkhoven R, Vrancken Peeters MJ, Sonke GS, Rodenhuis S, Wessels LF: SERPINA6, BEX1, AGTR1, SLC26A3, and LAPTM4B are markers of resistance to neoadjuvant chemotherapy in HER2-negative breast cancer. Breast Cancer Res Treat 2013;137:213-223.

17 Ateeq B, Tomlins SA, Chinnaiyan AM: AGTR1 as a therapeutic target in ER-positive and ERBB2-negative breast cancer cases. Cell Cycle 2009;8:3794-3795. 


\section{Cellular Physiology Cell Physiol Biochem 2018;45:1581-1589

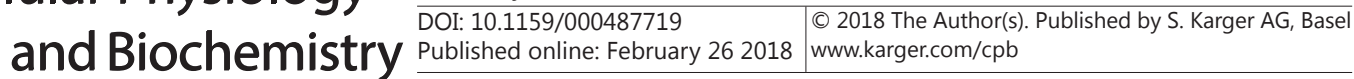 \\ Zhao et al.: Modulation of OS Chemo-Sensitivity}

18 Rhodes DR, Ateeq B, Cao Q Tomlins SA, Mehra R, Laxman B, Kalyana-Sundaram S, Lonigro RJ, Helgeson BE, Bhojani MS, Rehemtulla A, Kleer CG, Hayes DF, Lucas PC, Varambally S, Chinnaiyan AM: AGTR1 overexpression defines a subset of breast cancer and confers sensitivity to losartan, an AGTR1 antagonist. Proc Natl Acad Sci U S A 2009;106:10284-10289.

19 Pu Y, Zhao F, Li Y, Cui M, Wang H, Meng X, Cai S: The miR-34a-5p promotes the multi-chemoresistance of osteosarcoma via repression of the AGTR1 gene. BMC Cancer 2017;17:45.

-20 Fan L, Zhu C, Qiu R, Zan P, Zheng Z, Xu T, Li G: MicroRNA-661 Enhances TRAIL or STS Induced Osteosarcoma Cell Apoptosis by Modulating the Expression of Cytochrome c1. Cell Physiol Biochem 2017;41:1935-1946.

-21 Tian X, Zhang X: A Single Nucleotide Polymorphism (rs1056629) in 3'-UTR of MMP-9 is Responsible for a Decreased Risk of Metastatic Osteosarcoma by Compromising its Interaction with microRNA-491-5p. Cell Physiol Biochem 2016;38:1415-1424.

22 Chen G, Fang T, Huang Z, Qi Y, Du S, Di T, Lei Z, Zhang X, Yan W: MicroRNA-133a Inhibits Osteosarcoma Cells Proliferation and Invasion via Targeting IGF-1R. Cell Physiol Biochem 2016;38:598-608.

23 Chen B, Huang Z, Zhang Y, Chen Y, Li Z: MicroRNA-145 Suppresses Osteosarcoma Metastasis via Targeting MMP16. Cell Physiol Biochem 2015;37:2183-2193.

24 Shao XJ, Miao MH, Xue J, Xue J, Ji XQ, Zhu H: The Down-Regulation of MicroRNA-497 Contributes to Cell Growth and Cisplatin Resistance Through PI3K/Akt Pathway in Osteosarcoma. Cell Physiol Biochem 2015;36:2051-2062. 